\title{
Investigation of Defects in 2D Perovskite Oxide Nanosheets
}

\section{Ceren Yilmaz Akkaya ${ }^{1}$, Tugba Isik ${ }^{2},{\text { Haiyan } \mathrm{Tan}^{3}, \text { Ugur Unal }^{4} \text { and Volkan Ortalan }}^{5}$}

${ }^{1}$ Department of Materials Science and Engineering, University of Connecticut, Storrs, Connecticut, United States, ${ }^{2}$ School of Mechanical Engineering, Purdue University, United States, ${ }^{3}$ Centre for Advanced Microscopy and Materials Analysis (CAMMA), University of Connecticut, United States, ${ }^{4}$ Chemistry Department, Koc University, Istanbul, Turkey, ${ }^{5}$ Department of Materials Science and Engineering, University of Connecticut, United States

Layered perovskite oxides, have been attracting interest widely due to their rich compositional variety and flexible interlayer galleries that facilitates chemical modification. Especially, 2D oxide nanosheets derived from the layered perovskites via exfoliation reactions have been at the center of this attention. 2D complex oxides display superior electronic, optical, mechanical properties providing a remarkable toolbox for designing multifunctional materials with critical thicknesses. Some of the most promising application fields are (photo)catalysis, optoelectronics, energy conversion and storage, electrochromic devices, (bio) chemical sensing, and nanoelectronics.1-5

The parent layered compounds are composed of $n$ repeating layers of (2D)-ABO3 slabs separated by cations or cationic units with $n=\infty$ corresponding to the cubic perovskite structure. Ease of modification of the A and B site cations, ions in the interlayer gallery as well as the number of perovskite layers (n) provide layered perovskites and their corresponding nanosheets with great structural diversity and functionality. In particular, electronic and photocatalytic properties of nanosheets can be tailored by doping in the A and/or B site even in minute amounts, which provides control on the connectivity/rotation of the octahedron units.6-8

High-angle annular dark-field scanning transmission electron microscopy (HAADF-STEM) is a remarkably powerful technique for real-space imaging at sub-angstrom resolution with $\mathrm{Z}$-contrast $(\mathrm{Z}=$ atomic number). The HAADF image intensity is approximately proportional to (Z2) 9 with heavy atoms appearing brighter which allows identification of the atomic species through intensity patterns in the image. Additionally, by performing electron energy loss spectroscopy (EELS) in combination with the Z-contrast imaging, atomic resolution information on chemistry, structure, and electronic properties for complex material systems can be obtained.10 Hence, this method provides great path for analysis of chemical and electronic structure of materials with single-atom sensitivity.10,11

Detailed understanding of the atomic structure of the modified structure is needed to correlate the local structural changes with physical behavior of materials. However, evaluation of structural properties of nanosheets of molecular thicknesses, exact position of the chemical substitutions and defect sites created by doping is challenging through conventional techniques. Although there has been effort to investigate the atomic structure of 2D oxide nanosheets by STEM analysis 12, detailed investigation on the dopant induced changes in $2 \mathrm{D}$ oxide structure is still needed.

In this work, we focus on calcium niobate $(\mathrm{KCa} 2 \mathrm{Nan}-3 \mathrm{NbnO} 3 \mathrm{n}+1)$ and investigate the effect of introduction of aliovalent substitution in the $\mathrm{Ca} 2+$ sites in structural and electronic properties of the bulk and 2D nanomaterials. The powders are prepared by solid state or sol-gel synthesis and nanosheets are derived by soft chemical exfoliation (Figure 1). The products are analysed by aberration corrected STEM/EELS (TitanTM Themis). The effect of dopant concentration on the structural, optical, and photocatalytic properties of the perovskite oxides will be discussed. 

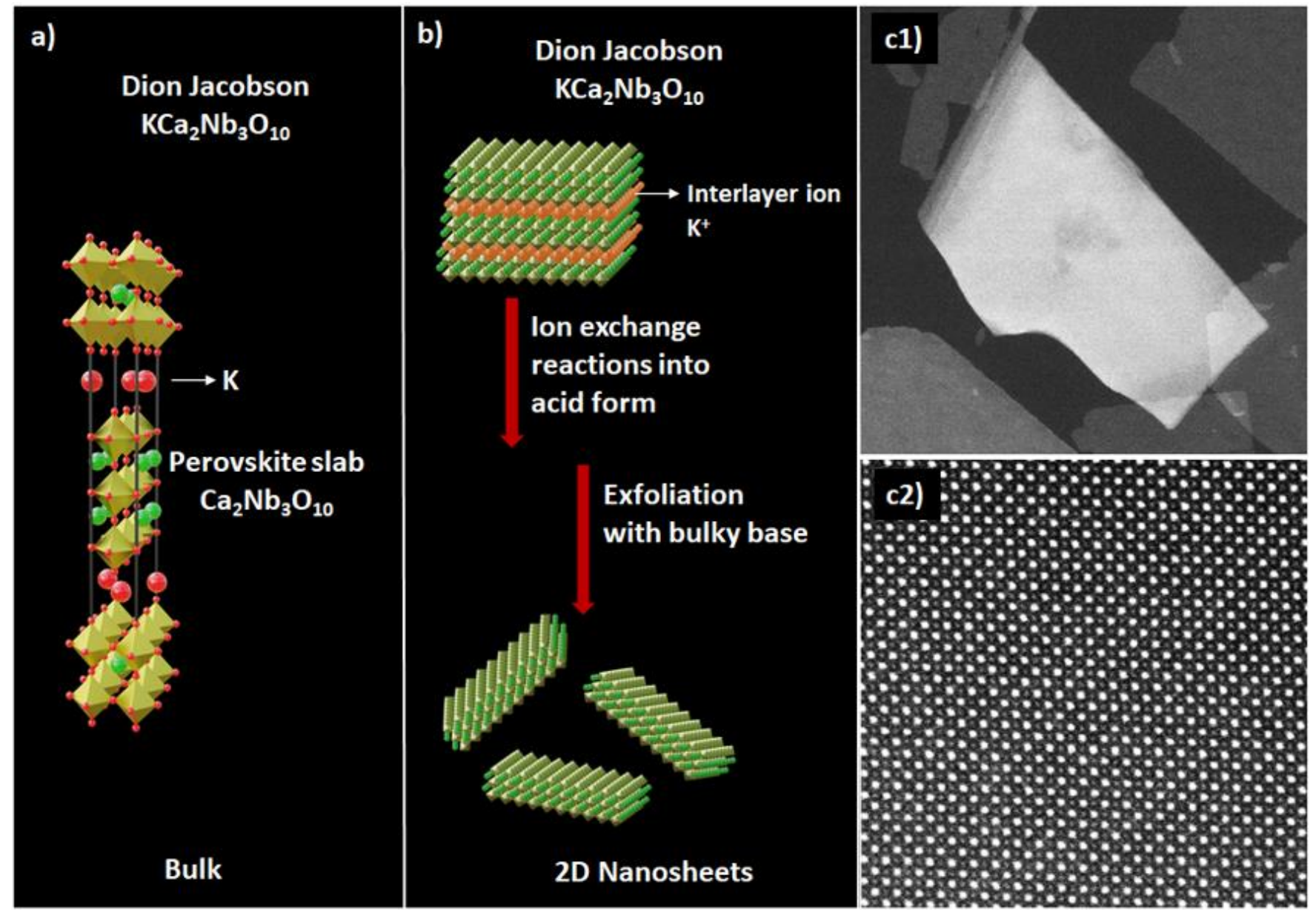

Figure 1. Schematic representation of a) structure of Dion-Jacobson phase layered perovskite oxide, b) soft synthesis of 2D sheets from 3D parent material via exfoliation c1-2) HAADF-STEM images of Ru doped $\mathrm{Ca} 2 \mathrm{Nb} 3 \mathrm{O} 10$ nanosheets

\section{References}

1. Timmerman, M. A., Xia, R., Le, P. T. P., Wang, Y. \& Elshof, J. E. Metal Oxide Nanosheets as 2D Building Blocks for the Design of Novel Materials. Chem. - A Eur. J. 26, 9084-9098 (2020).

2. Kim, Y. H. et al. 2D perovskite nanosheets with thermally-stable high- $\kappa$ response: A new platform for hightemperature capacitors. ACS Appl. Mater. Interfaces 6, 19510-19514 (2014).

3. Osada, M. \& Sasaki, T. The rise of 2D dielectrics/ferroelectrics. APL Mater. 7, (2019).

4. Unal, U. et al. Visible light photoelectrochemical activity of $\mathrm{K}_{4} \mathrm{Nb}_{6} \mathrm{O}_{17}$ intercalated with photoactive complexes by electrostatic self-assembly deposition. J. Solid State Chem. 179, 33-40 (2006).

5. Sarac, F. E., Yilmaz, C., Acar, F. Y. \& Unal, U. CdTe quantum dot sensitized hexaniobate nanoscrolls and their photoelectrochemical properties. RSC Adv. 2, 10182-10184 (2012).

6. Li, L., Xie, L. \& Pan, X. Real-time studies of ferroelectric domain switching: A review. Reports Prog. Phys. $82,(2019)$.

7. Jo, H., Oh, S. \& Ok, K. M. Noncentrosymmetric (NCS) solid solutions: elucidating the structure-nonlinear optical (NLO) property relationship and beyond. Dalton 46, 15628-15635 (2017).

8. Li, B. W. et al. A-site-modified perovskite nanosheets and their integration into high- $\kappa$ dielectric thin films with a clean interface. Jpn. J. Appl. Phys. 49, 3-8 (2010)

9. Pennycook, S. J., Kirkland, A. I., Varela, M. \& Hetherington, C. J. D. Materials Advances through Aberration- Corrected Electron. MRS Bull. 31, 36-43 (2006). 
10. Nellist, P. D. \& Pennycook, S. J. Direct Imaging of the Atomic Configuration of Ultradispersed Catalysts. Science (80-. ). 274, 413-415 (1996).

11. Ortalan, V., Uzun, A., Gates, B. C. \& Browning, N. D. Direct imaging of single metal atoms and clusters in the pores of dealuminated HY zeolite. Nat. Nanotechnol. 5, 506-510 (2010).

12. Choi, H. et al. Atomic Doping Site and Dielectric Property in Perovskite Oxide Nanosheets. Microsc. Microanal. 25, 2152-2153 (2019). 